\title{
Hybrid Organic/Inorganic Materials Based on Functionalized Dendritic Polymers: Methods of Preparation, Applications and Future Prospects
}

\section{Michael Arkas*}

Institute of Physical Chemistry, NCSR Demokritos, Greece

Dendritic polymers [1-6] is a special class of macromolecules consisting of monomeric units branching out from a common centre. The name dendritic is derived from the Greek word "Dendritic" meaning "tree-like". They consist of four subcategories which correspond to different topologies:

i) dendrimers: perfectly symmetric macromolecules with precisely fixed number of monomeric units

ii) hyperbranched polymers: non-symmetrical, polydisperse, randomly polymerized macromolecules

iii) dendrons: monodisperse wedge-shaped dendrimer sections

iv) dendrigrafts: dendritic structures that are grown on reactive linear polymers or oligomers

The distinctive highly branched chemical structure of dendritic polymers has nanosized dimensions and consists of the central core, the repeating units and the terminal functional groups. Due to these structural features, they possess nanocavities that are able to encapsulate various molecules, while their external surfaces can be easily functionalized. In addition, their terminal groups can exhibit the so-called polyvalency effect $[7,8]$, which enhances their binding with other substrates.

The diversity of dendritic polymers nanocavities as far as the size, polarity and interaction ability are concerned, provides the basis for the formation of devices bearing nanosized containers for effectively encapsulating metal ions. Thus, colloidal silver nanoparticles are obtained by the reduction Ag+ ions encapsulated into Polyethylene Imine (PEI) Hyperbranched Polymer even in the absence of another reductant [9]. Dendritic polymers also stabilize colloidal suspensions of gold by in situ reduction of $\mathrm{HAuCl}_{4}$ encapsulated into Polyamidoamine PAMAM Dendrimers by aqueous $\mathrm{NaBH}_{4}$ solution [10]. These dendritic polymer/noble metal composites have unique potential for applications such as catalysis.

On the other hand the terminal functional groups can be involved in a variety of interactions/modifications. For example the amines of the poly (propyleneimine) and poly (amidoamine) dendrimers and hyperbranched polymers act as templates for biomimetic silica nanospheres formation [11]. The sol-gel reactions proceed at room temperature by the addition of metastable silicic acid into the dendrimer solution in phosphate buffer, $\mathrm{pH}$ 7.5. The dendritic polymers, although incorporated in the nanospheres, retain their property of encapsulating metal ions and therefore, can be applied for catalysis. In addition a variety of other compounds can be encapsulated depending on the microenvironment of the nanocavities, which is primarily affected by the structural characteristics of the repeating units. In this manner, encapsulation of impurities dissolved in water leads, using appropriate methods, to novel bulk water purification technologies [12] and the solubilisation of bioactive compounds in their interior could lead to the development of effective drug and gene delivery systems [13].

The reactivity of the functionalized terminal groups of the dendritic polymers can result in chemical bond formation with different substrates. In this manner covalent attachment of a polymer with desired properties to a solid support can be achieved. For example by employing ethoxysilylated derivatives of diaminobutane poly (propylene imine) and polyglycerol by hydrolysis and subsequent crosslinking nanostructured networks can be produced which interact with ceramic filters, forming a covalently bound film that has the potential to encapsulate pollutants of water through continuous filtration.

In a combination of the two above mentioned methods crosslinking of hyperbranched macromolecules functionalized with silica precursors along with hydrolysis and poly-condensation reactions leads to the formation of novel silica network architecture. In this case the solid support is synthesized by the sol gel reactions of the terminal groups.

Another approach for the production of hybrid dendritic/inorganic materials is the direct propagation of dendrons on the surface of inorganic materials such as silica gel through solid-phase synthesis via a conventional divergent pathway. For example the propagation of PAMAM (polyamidoamine) dendrons on silica can be accomplished by a series of alternate Michael addition and amidation reactions [14]. PAMAM modified silica is further functionalized with long aliphatic chains to the periphery of the dendron for applications in pollutant encapsulation. PAMAM dendritic polymers are also phosphonated in order to provide an attractive coordination site for rhodium. The resulting complexes are used for the catalytic hydroformylation of olefins. Another modification of the periphery of PAMAM dendrons by salicylaldehyde affords a substrate that immobilizes $\mathrm{Mn}$ (II) onto PAMAM-SiO ${ }_{2}$ dendrimers for the preparation of catalysts for olefin epoxidation.

In another aspect, by combining the chemical attachment of dendritic polymers to a solid support with biomimetic synthesis, a hydroxyapatite layer, with characteristics similar to those of biogenic apatite, firmly attached on the titanium surface is produced. This approach includes titanium surface activation by proper chemical pretreatment, chemical bonding with an appropriately functionalized siloxane polymer intermediate layer and subsequent chemical attachment of hyperbranched poly(ethylene imine) (PEI) that is able to promote the formation, of a firmly attached calcium phosphate layer [15]. These composites have potential applications in manufacturing dental implants and in orthopedics for bone-anchoring systems.

*Corresponding author: Michael Arkas, Institute of Physical Chemistry, NCSR Demokritos, Aghia Paraskevi 15310 Attiki, Greece, E-mail: markas@chem.demokritos.gr

Received August 23, 2012; Accepted August 24, 2012; Published August 31, 2012

Citation: Arkas M (2012) Hybrid Organic/Inorganic Materials Based on Functionalized Dendritic Polymers: Methods of Preparation, Applications and Future Prospects. J Material Sci Eng 1:e103. doi:10.4172/2169-0022.1000e103

Copyright: (c) 2012 Arkas M. This is an open-access article distributed under the terms of the Creative Commons Attribution License, which permits unrestricted use, distribution, and reproduction in any medium, provided the original author and source are credited. 
Citation: Arkas M (2012) Hybrid Organic/Inorganic Materials Based on Functionalized Dendritic Polymers: Methods of Preparation, Applications and Future Prospects. J Material Sci Eng 1:e103. doi:10.4172/2169-0022.1000e103

Page 2 of 2

The manufacturing of hybrid dendritic-inorganic materials can thus be achieved by employing several convenient methods and techniques including, chemical reactions such as sol-gel and biomimetic synthesis, covalent attachment of a dendritic compound to a inorganic support direct propagation of a dendron from the inorganic matrix or encapsulation of metal ions into the dendritic polymers. These materials receive very high scientific interest since they have many other potential applications. For example dendritic polymers produce a vast variety of differently colored metal complexes, property which is preserved in the hybrid materials as well. Possible applications for the detection of explosives, chemical warfare agents, and other substances even in small concentrations can be investigated. The use of biogenic silica produced by a complex of DNA with a dendrimer could simplify DNA transfection process. Hybrid nanoceramics derived from silica, alumina, zirconia and titania and dendritic polymers can be tested as drug-delivery vehicles because of their inert nature and high compatibility with biological systems. It is evident that the formation of composite devices which will have extended applications for specific cases can be accomplished by careful selection of the suitable materials and processing parameters. It is thus hoped that the work performed and reviewed in this editorial will show the potential of the methodology and will trigger interest to other researchers to further contribute and exploit this promising area of research.

\section{References}

1. Vogtle F, Gestermann S, Hesse R, Schwierz H, Windisch B (2000) Functional dendrimers. Prog Polym Sci 25: 987-1047.

2. Frechet JMJ, Tomalia DA (2001) Dendrimers and Other Dendritic Polymers. $1^{\text {st }}$ Edn J Wiley \& Sons Ltd Chichester, UK.

3. Newkome GR, Moorefield CN, Vogtle F (2001) Dendrimers and Dendrons: Concepts, Syntheses, Perspectives. $1^{\text {st }}$ edn Wiley-VCH Weinheim, Germany.
4. Svenson S, Tomalia DA (2005) Dendrimers in biomedical applications reflections on the field. Adv Drug Deliv Rev 57: 2106-2129.

5. Lee CC, MacKay JA, Frechet JMJ, Szoka FC (2005) Designing dendrimers for biological applications. Nat Biotechnol 23: 1517-1526.

6. Newkome GR, Shreiner CD (2008) Poly(amidoamine), polypropylenimine, and related dendrimers and dendrons possessing different $1 \rightarrow 2$ branching motifs: An overview of the divergent procedures. Polymer 49: 1-173.

7. Mammen M, Choi SK, Whitesides GM (1998) Polyvalent Interactions in Biological Systems: Implications for Design and use of Multivalent Ligands and Inhibitors. Angew Chem Int Edit 37: 2754-2794.

8. Kitov PI, Bundle DR (2003) On the nature of the multivalency effect: a thermodynamic model. J Am Chem Soc 125: 16271-16284.

9. Manoth M, Manzoor K, Patra MK, Pandey P, Vadera SR et al. (2009) Dendrigraft polymer-based synthesis of silver nanoparticles showing bright blue fluorescence. Mater Res Bull 44: 714-717.

10. Garcia ME, Baker LA, Crooks RM (1999) Preparation and characterization of dendrimer-gold colloid nanocomposites. Anal Chem 71: 256-258.

11. Knecht MR, Wright DW (2004) Amine-Terminated Dendrimers as Biomimetic Templates for Silica Nanosphere Formation. Langmuir 20: 4728-4732.

12. Arkas M, Tsiourvas D (2009) Organic/inorganic hybrid nanospheres based on hyperbranched poly(ethylene imine) encapsulated into silica for the sorption of toxic metal ions and polycyclic aromatic hydrocarbons from water. J Hazard Mater 170: 35-42.

13. Kolhe P, Misra E, Kannan RM, Kannan S, Lieh-Lai M (2003) Drug complexation, in vitro release and cellular entry of dendrimers and hyperbranched polymers. Int J Pharm 259: 143-160.

14. Tsubokawa N, Ichioka H, Satoh T, Hayashi S, Fujiki K (1998) Grafting of 'dendrimer-like' highly branched polymer onto ultrafine silica surface. React Funct Polym 37: 75-82.

15. Tsiourvas D, Tsetsekou A, Arkas M, Diplas S, Mastrogianni E (2011) Covalent attachment of a bioactive hyperbranched polymeric layer to titanium surface for the biomimetic growth of calcium phosphates. J Mater Sci Mater 22: 85-96. 